\title{
Oat and lipolysis: food matrix effect
}

Article

Accepted Version

Wilde, P. J., Garcia-Llatas, G., Lagarda, M. J., Haslam, R. P. and Grundy, M. M. L. (2019) Oat and lipolysis: food matrix effect. Food Chemistry, 278. pp. 683-691. ISSN 0308-8146 doi: https://doi.org/10.1016/j.foodchem.2018.11.113 Available at https://centaur.reading.ac.uk/80772/

It is advisable to refer to the publisher's version if you intend to cite from the work. See Guidance on citing.

To link to this article DOI: http://dx.doi.org/10.1016/j.foodchem.2018.11.113

Publisher: Elsevier

All outputs in CentAUR are protected by Intellectual Property Rights law, including copyright law. Copyright and IPR is retained by the creators or other copyright holders. Terms and conditions for use of this material are defined in the End User Agreement.

\section{$\underline{\text { www.reading.ac.uk/centaur }}$}

\section{CentAUR}

Central Archive at the University of Reading

Reading's research outputs online 


\section{Oat and lipolysis: Food matrix effect}

Peter J. Wilde ${ }^{\mathrm{a}}$, Guadalupe Garcia-Llatas ${ }^{\mathrm{b}}$, María Jesús Lagarda ${ }^{\mathrm{b}}$, Richard P. Haslamc, Myriam M.L. Grundy ${ }^{\text {ad* }}$

${ }^{a}$ Quadram Institute Bioscience, Norwich Research Park, Colney, Norwich NR4 7UA, UK

${ }^{b}$ Nutrition and Food Science Area, Faculty of Pharmacy, University of Valencia, Avda.

Vicente Andrés Estellés s/n, 46100 Burjassot (Valencia), Spain

${ }^{\mathrm{c}}$ Rothamsted Research, Department of Plant Sciences, Harpenden, AL5 2JQ, UK

${ }^{\mathrm{d}}$ School of Agriculture, Policy and Development, Sustainable Agriculture and Food Systems

Division, University of Reading, Earley Gate, Reading, RG6 6AR, UK

peter.wilde@quadram.ac.uk, guadalupe.garcia@uv.es, m.j.lagarda@uv.es,

richard.haslam@rothamsted.ac.uk, m.m.grundy@reading.ac.uk

*Corresponding author: Myriam M.L. Grundy, School of Agriculture, Policy and Development, Sustainable Agriculture and Food Systems Division, University of Reading, Reading, RG6 6AR, UK. Tel.: +44 01183 877868, m.m.grundy@ reading.ac.uk 


\section{ABSTRACT}

Oat is rich in a wide range of phytochemicals with various physico-chemical,

3 colloidal and interfacial properties. These characteristics are likely to influence human lipid

4 metabolism and the subsequent effect on health following oat consumption. The aim of this

5 work was to investigate the impact of oat materials varying in complexity on the lipolysis

6 process. The composition, structure and digestibility of different lipid systems (emulsions, oil

7 bodies and oil enriched in phytosterols) were determined. The surface activities of

8 phytosterols were examined using the pendant drop technique. Differences in lipid

9 digestibility of the oat oil emulsions and the oil bodies were clearly seen. Also, the digestion

10 of sunflower oil was reduced proportionally to the concentration of phytosterols present. This

11 may be due to their interfacial properties as demonstrated by the pendant drop experiments.

12 This work highlights the importance of considering the overall the-structure of the system

13 studied and not only its composition.

14

15 Keywords: Oat lipid, food matrix, lipolysis, phytosterols, interface, micelles.

16

17 Abbreviations: FFA, free fatty acids; Ocrude, crude oil from oats; OPL4, oat oil with 4\%

18 polar lipids; OPL15, oat oil with 15\% polar lipids; PS, phytosterols; SOs, Sunflower oil

19 treated with Florisil®; WPI, whey protein isolate.

20

21 Chemical compounds studied in this article 
$22 \beta$-sitosterol (PubChem CID: 222284); Campesterol (PubChem CID: 173183); $\Delta$ 5-avenasterol 23 (PubChem CID: 5281326); $\Delta$ 7-avenasterol (PubChem CID: 12795736);

24 Digalactosyldiacylglycerol (PubChem CID: 25203017); Epicoprostanol (PubChem CID:

25 91465); Fucosterol (PubChem CID: 5281328); Monogalactosyldiacylglycerol (PubChem

26 CID: 25245664); Phosphatidylcholine (PubChem CID: 6441487); Stigmasterol (PubChem

27 CID: 5280794). 


\section{7}

\section{Introduction}

The association between oat and its positive effect on human lipid metabolism, in particular decreases in blood cholesterol levels, has been extensively investigated in vivo (Thies, Masson, Boffetta, \& Kris-Etherton, 2014). Several mechanisms of action have been proposed linked to the $\beta$-glucan contained in oat (Wolever et al., 2010), which includes an increase in viscosity of intestinal contents and interaction with bile salts leading to restricted bile salts recycling (Gunness \& Gidley, 2010). However, it is likely that the observed benefits on health are also due to other structural features unique to the oat grain that would dictate the way it behaves during digestion. For instance, our recent in vitro data suggests that oat compounds, other than $\beta$-glucan, may have an impact on lipid digestibility (Grundy et al., 2017; Grundy, McClements, Ballance, \& Wilde, 2018). Oat is rich in macronutrients and bioactives phytochemicals, including arabinoxylans, antioxidants (e.g. phenolic acids, avenanthramides, tocotrienols, and saponins) and phytosterols (Shewry et al., 2008; Welch, 2011). Among those constituents, another potential contributor to the positive effect of oat consumption on plasma cholesterol levels could be the phytosterols (Bard, Paillard, \& Lecerf, 2015). Similarly to $\beta$-glucan, the exact processes behind this effect remain unclear, although their interaction with the absorption of cholesterol by displacement of cholesterol from the mixed micelles and formation of mixed crystals leading to cholesterols precipitation and excretion is currently the strongest explanation (De Smet, Mensink, \& Plat, 2012).

Phytosterols are often studied as isolated compounds, however plant-based foods such as oats are mostly in the form of complex matrices whose constituents interact with each other. The food matrix has been demonstrated to be an important parameter to influence the play a significant role in the functionality of phytosterols (Cusack, Fernandez, \& Volek, 2013;

Gleize, Nowicki, Daval, Koutnikova, \& Borel, 2016). The forms that in which they are 
53 delivered to the gastrointestinal tract is probably a crucial element to their bioactivity. Indeed,

54 phytosterol bioavailability and efficacy has been shown to rely on many factors such as the

55 type and quantity of lipids present, the type of phytosterols (i.e. free, esters or steryl

56 glycosides sterel, stanel or ester such as steryl glycoside), the source, and the food

57 microstructure (Ostlund, 2002; Alvarez-Sala et al., 2016; Ferguson, Stojanovski, MacDonald-

58 Wicks, \& Garg, 2016). In plant-based foods, phytosterols are found as a mixture of free and

59 bound (esters) phytosterols, but not all forms have the same physico-chemical properties and

60 therefore health benefits (Moreau, Whitaker, \& Hicks, 2002). Furthermore, phytosterols are

61 hydrophobic and poorly soluble in aqueous solutions, so they associate, mainly via

62 hydrophobic interactions, with various lipophilic structures that are present during digestion,

63 such as oil droplets, lipid vesicles, membranes, and micelles, and this is thought to be critical

64 for their functionality (Piironen, Lindsay, Miettinen, Toivo, \& Lampi, 2000; Amiot et al., 65 2011).

66

67

Some studies have investigated the fate of the phytosterols during in vitro lipid

68 digestion (von Bonsdorff-Nikander et al., 2005; Moran-Valero, Martin, Torrelo, Reglero, \&

69 Torres, 2012; Zhao, Gershkovich, \& Wasan, 2012; Alvarez-Sala et al., 2016; Gleize, Nowicki,

70 Daval, Koutnikova, \& Borel, 2016), but mechanistic studies that could provide information

71 about how they influence lipolysis and micelles formation using different oat matrices are

72 missing. To improve our understanding gain insight into the role played by the broader oat

73 matrix composition and structure on lipid digestion, in this work, we examined various

74 aspects of lipolysis were examined, focusing on the contribution made by phytosterols. We

75 believe that The kinetics of lipolysis and the mixed micelle formation have important

76 consequences on lipid and cholesterol uptake. During digestion, complex, dynamic self-

77 assembly of amphiphilic and lipophilic molecules occurs, which governs the nature and fate 
78 (absorption) of the lipophilic molecules (Phan, Salentinig, Prestidge, \& Boyd, 2014). Our The

79 hypothesis of this study was that the oat matrix structure would affect the bioaccessibility and

80 behaviour in solution of the phytosterols and thereby impact lipid digestibility and the

81 formation of mixed micelles. To test this hypothesis, menitered the lipolysis kinetics of a

82 range of materials with different degrees of complexity was monitored using the pH-stat

83 method. The mixed micelles generated were analysed for particle size and charge. Finally, the

84 effect of phytosterols on the interfacial tension of sunflower oil was also examined using the

85 pendant drop technique.

86

87 2. Materials and Methods

88 2.1. Materials

89 Oat groats (Avena sativa L.; variety Belinda) were obtained from Lantmännen

90 Cerealia, Moss, Norway. Oat oils of different purities (OPL4 and OPL15, containing

91 approximately 4 and $15 \%$ of polar lipids, respectively; and crude oat oil, Ocrude) were a

92 generous gift from Swedish Oat Fiber (Swedish Oat Fiber AB, Bua, Sweden). Sunflower oil,

$93 \beta$-sitosterol (70\% purity), epicoprostanol ( $5 \beta$-cholestan-3 $\alpha$-ol, $95 \%$ purity; used as internal

94 standard), $\beta$-sitosterol (95\% purity), stigmasterol (95\% purity), fucosterol ( $93 \%$ purity),

95 pancreatin (40 U/mg of solid based on lipase activity), bovine bile extract, sodium

96 taurocholate (NaTC, 97\%), sodium glycodeoxycholate (NaGDC, 97\%), sodium dihydrogen

97 phosphate (99\%), disodium hydrogen phosphate (99\%), sodium chloride (99.8\%), calcium

98 chloride (99\%), potassium hydroxide (99.97\%), N,O-Bis(trimethylsilyl)trifluoroacetamide

99 with trimethylchlorosilane (BSTFA+1\% TMCS) were purchased from Sigma (Poole, UK).

100 The internal standards (phosphatidylcholine, PC, phosphatidylethanolamine, PE,

101 phosphatidylinositol, PI, phosphatidylglycerol, PG, lysophosphatidylcoline, lysoPC,

102 digalactosyldiacylglycerol and monogalactosyldiacylglycerol) for phospholipids and 
galactolipids analysis were supplied by Avanti (Alabama, USA). Pyridine, extra dry (99.5\%)

104 was obtained from Fisher Scientific (Loughborough, UK). Campesterol (98\% purity), $\Delta 5$ 105 avenasterol (98\% purity) and $\Delta 7$-avenasterol (98\% purity) were obtained from ChemFaces

106 (Wuhan, China). Powdered whey protein isolate (WPI) was donated by Davisco Foods 107 International (Le Sueur, USA).

\subsection{Material preparation}

\subsubsection{Oat oil bodies}

Oat groats were ground in a coffee grinder (F20342, Krups, Windsor, UK) and soaked

112 overnight in extraction media (1:5, w/v; $10 \mathrm{mM}$ sodium phosphate buffer $\mathrm{pH} 7.5,0.6 \mathrm{M}$

113 sucrose) as previously described (White, Fisk, \& Gray, 2006). The soaked oats were

114 homogenised (Laboratory blender 8010ES, Waring Commercial, USA) at full power for 2

115 min and the slurry filtered through 3 layers of cheesecloth to remove large particles and cell

116 fragments. The filtrate was then centrifuged (Beckman J2-21 centrifuge; fixed rotor JA-10) at

$11720000 \mathrm{~g}, 4^{\circ} \mathrm{C}$ for $20 \mathrm{~min}$. The creamy upper layer was recovered, this is referred to as the oil

118 bodies. The sucrose added to the extraction media facilitated the separation of oil bodies from

119 the rest of the oat constituents (e.g. starch and storage proteins) as it allowed them to float on 120 top of the solution following filtration and centrifugation.

Sunflower oil (SOs) was treated with Florisil@ (Sigma, Poole, UK), which is a porous and absorbent form of magnesium silicate, used to remove polar, surface-active compounds 125 (e.g. phospholipids, galactolipids and sterols) from the oil. Sunflower oil enriched in 126 phytosterols was obtained by mixing the Florisil@-treated sunflower oil with the $\beta$-sitosterol 127 from Sigma (70\% purity; final phytosterol concentration of 0, 0.5, 1.0, 1.5 and 2.0\%) based 
128 on a method by Mel'nikov et al. 2004 . The mixture was heated at $75^{\circ} \mathrm{C}$ during 15 min under

129 intensive stirring until complete dissolution of the crystalline phase. The solution was cooled 130 down to $25^{\circ} \mathrm{C}$ for $100 \mathrm{~min}$ using a water bath. The oils enriched in phytosterols were used

131 within 5 days to prevent the formation of sterol crystals (checked by light microscopy, data 132 not shown).

\subsubsection{Emulsions}

The emulsions were prepared as described in a previous study (Grundy et al., 2017).

136 Briefly, WPI solution was prepared by dissolving $1 \mathrm{wt} \%$ of powdered WPI into $10 \mathrm{mM}$

137 phosphate buffer ( $\mathrm{pH} 7.0$ at $37^{\circ} \mathrm{C}$ ) and stirring for at least $2 \mathrm{~h}$. Emulsions were made from

138 either oat oils (Ocrude, OPL15, and OPL4), or Florisil@-treated sunflower oil with or without 139 phytosterols. The emulsions were obtained by pre-emulsifying $1.6 \mathrm{wt} \%$ of oil in WPI solution 140 using a homogeniser (Ultra-Turrax T25, IKA® Werke, from Fisher Scientific Ltd.) for 1 min 141 at $1100 \mathrm{rpm}$. The pre-emulsion was then sonicated with an ultrasonic processor (Sonics \& 142 Materials Inc, Newtown, Connecticut, USA) at 70\% amplitude for 2 min.

\subsection{Characterisation of the material}

Moisture content was determined by weighing $200 \mathrm{mg}$ of oat bodies or oil into

146 microtubes that were placed in a vacuum oven (Townson \& Mercer Ltd, Stretford, Greater

147 Manchester, $\mathrm{UK}$ ) at $40^{\circ} \mathrm{C}$ for $48 \mathrm{~h}$. The dried samples were then weighed a second time and

148 the moisture content calculated by difference.

Total lipid content of the materials was obtained by Folch extraction, fatty acid methyl esters (FAME) derivatisation and Gas Chromatography-Mass Spectrometry (GC-MS; Agilent

151 7890B/5977A GC/MSD, Agilent Technologies, Santa Clara, California, USA) analysis

152 (Grundy et al., 2017). 
Phytosterol content was determined by a method adapted from a previous study

154 (Alvarez-Sala et al., 2016). Briefly, hot saponification was performed on $100 \mathrm{mg}$ of samples with $1 \mathrm{~mL} 2 \mathrm{~N} \mathrm{KOH}$ in ethanol/water $\left(9: 1, \mathrm{v} / \mathrm{v} ; 65^{\circ} \mathrm{C}\right.$ during $\left.1 \mathrm{~h}\right)$, followed by extraction of

156 the unsaponifiable fraction with diethyl ether and derivatisation with BSTFA $+1 \%$

157 TMCS/pyridine (10:3, v/v). The BSTFA derivatives were dissolved in $100 \mu \mathrm{L}$ of $n$-hexane 158 and analysed by GC. One $\mu \mathrm{L}$ of sample was injected in the GC equipped with a CP-Sil 8 low 159 bleed/MS (50 m, $0.25 \mathrm{~mm}, 0.25 \mu \mathrm{m}$ ) capillary column (Agilent Technologies, Santa Clara, 160 USA). The oven was initially programmed at $150^{\circ} \mathrm{C}$, maintained during $3 \mathrm{~min}$, heated to $161280^{\circ} \mathrm{C}$ at a rate of $30^{\circ} \mathrm{C} / \mathrm{min}$, and kept during $28 \mathrm{~min}$, then raised to $295^{\circ} \mathrm{C}$ at a rate of

$16210^{\circ} \mathrm{C} / \mathrm{min}$. Finally, this temperature was maintained for $10 \mathrm{~min}$. The carrier gas was helium

163 (15 psi). The temperature of both the injector port and the flame ionisation detector were $164325^{\circ} \mathrm{C}$, and a pulsed split ratio of 1:10 was applied. Quantitative analyses of the polar lipids, i.e., phospholipids and galactolipids (phosphatidylcholine, phosphatidylethanolamine, phosphatidylinositel, phosphatidylglycerol, lysophosphatidylcoline, digalactosyldiglycerol or monogalactesyldiglycerol), were carried out using electrospray ionization tandem triple quadrupole mass spectrometry (API 4000; Applied

169 Biosystems; ESI-MS/MS). The lipid extractions were infused at $15 \mu \mathrm{L} / \mathrm{min}$ with an 170 autosampler (HTS-xt PAL, CTC-PAL Analytics AG, Switzerland). Data acquisition and acyl 171 group identification of the polar lipids were as described in Ruiz-Lopez et al. 2014 with 172 modifications. The internal standards for polar lipids were incorporated as: $0.857 \mathrm{nmol}$ 13:0173 LysoPC, 0.086 nmol di24:1-PC, 0.080 nmol di14:0-PE, 0.800 nmol di18:0-PI and 0.080 174 di14:0-PG. The standards and $10 \mu \mathrm{L}$ of sample were combined to make a final volume of 1 $175 \mathrm{~mL}$. 
179 a Beckman Coulter LS13320 ${ }^{\circledR}$ (Beckman Coulter Ltd., High Wycombe, UK). Water was used 180 as a dispersant (refractive index of 1.330), and the absorbance value of the oil droplets was 181 0.001. Crude oat oil had a refractive index of 1.463, OPL15 1.470, and OPL4 and sunflower 182 oil 1.473 as measured using a refractometer (Rhino Brix90 Handheld Refractometer,

183 Reichert, Inc., New York, USA). The particle size measurements were reported as the 184 surface-weighted mean diameter $\left(\mathrm{d}_{3,2}\right)$.

185 The micelles formed after $1 \mathrm{~h}$ digestion, with (digested) and without (blank) enzyme, 186 were obtained by centrifuging the digesta at $2200 \mathrm{~g}$ for $1 \mathrm{~h}$ at $10^{\circ} \mathrm{C}$ and filtrating the aqueous 187 fraction through $0.8 \mu \mathrm{m}$ and then $0.22 \mu \mathrm{m}$ filters (Gleize, Nowicki, Daval, Koutnikova, \& 188 Borel, 2016). The average size and zeta-potential of the micelles were determined with a 189 Zetasizer Nano-ZS (Malvern Instruments, Malvern, UK).

190 Values of particle size (volume or intensity percentage) are presented as the means \pm 191 SD of at least three replicates.

\subsection{In vitro duodenal digestion ( $p H$-stat)}

The rate and extent of lipolysis of oil bodies, oat oils (Ocrude, OPL15, and OPL4) and 195 sunflower oil containing various amounts of phytosterols were continuously measured by titration of released free fatty acids (FFA) with $0.1 \mathrm{M} \mathrm{NaOH}$ at $37^{\circ} \mathrm{C}$ and an endpoint of $\mathrm{pH}$

197 7.0. The details of the in vitro duodenal digestion model used can be found elsewhere 198 (Grundy et al., 2017). The final composition of the reaction system was $0.8 \mathrm{wt} \%$ lipid (300 $199 \mathrm{mg}$ of lipid from oil bodies or emulsion prepared as in Section 2.2.3.), $12.5 \mathrm{mM}$ bile salts, 2.4 $200 \mathrm{mg} / \mathrm{mL}$ lipase, $150 \mathrm{mM} \mathrm{NaCl}$ and $10 \mathrm{mM} \mathrm{CaCl}_{2}$. All lipolysis experiments were carried out in 201 triplicate. 


\subsection{Microstructural analysis}

The microstructure of the oat groats and oil bodies was studied using either optical

(Olympus BX60, Olympus, Southend-On-Sea, UK) or scanning electron (SEM; Zeiss Supra 55 VP FEG, Cambridge, UK) microscopes. For optical microscopy, samples of oil bodies at baseline, and before and after digestion, were stained with Nile red $(1 \mathrm{mg} / \mathrm{mL}$ in dimethyl sulfoxide) and then mounted on a glass slide, covered and viewed immediately. Oat groats observed by SEM were prepared as presented elsewhere (Grundy et al., 2017). 


\subsection{Statistical analysis}

The data were analysed using SPSS version 17.0. For all tests, the significance level was set at $p<0.05$ ( 2 tailed). The differences between the lipolysis of sunflower oil alone (SOs), and SOs enriched in phytosterols and the oat materials (i.e. oil bodies and oils) were analysed by one-way analysis of variance (ANOVA) followed by Dunnett's post-hoc test. $\zeta$-Potential of the micelles was analysed by one-way ANOVA followed by Tukey's post-hoc test and Student's paired $t$-test was used to evaluate differences between the blank and digested samples.

\section{Results and Discussion}

\subsection{Characterisation of the oat and sunflower materials}

Table 1 shows that the three oat oils were made of the same types of lipids (triacylglycerides, phospholipids, galactolipids and phytosterols), but differed in the proportion of these compounds. In particular, Ocrude and OPL15 contained about 14\% of phospholipids whereas OPL4 only 3.6\%. Galactolipids and phytosterols were found in much lower quantity than phospholipids, between 0.4 and $1.2 \mathrm{~g}$ in $100 \mathrm{~g}$ of oil for galactolipids and between 224 and $245 \mathrm{mg}$ in $100 \mathrm{~g}$ of oil for phytosterols. The oil bodies contained only $\sim 50 \mathrm{~g}$ of lipids for $100 \mathrm{~g}$ which is in agreement with a previous study (White, Fisk, \& Gray, 2006). Compared with the oat oils, oil bodies contained lower amount of phytosterols ( $\sim 230$ and 75 mg, for the oils and oil bodies, respectively), suggesting that the phytosterols are present mainly in the oil phase although a small amount may be embedded within the monolayers of phospholipids and proteins (oleosin) (Chen, Cao, Zhao, Kong, \& Hua, 2014). A significant proportion of the phytosterols present in the oils could have originated from the cell membranes when extracting the oil from the oat tissue (Hartmann, 1998). The phytosterols would have therefore existed in both esterified and free forms as they would have different 
253 physico-chemical properties and thereby they would partition between the membrane and the 254 oil phase (Moreau, Whitaker, \& Hicks, 2002).

255 The average droplets size of the emulsions made from the oat oils increased in the 256 following order: OPL4 $(2.0 \mu \mathrm{m})<\operatorname{SOs}(2.4 \mu \mathrm{m})<$ Ocrude $(3.4 \mu \mathrm{m})<$ oil bodies $(4.5 \mu \mathrm{m})<$ 257 OPL15 $(4.6 \mu \mathrm{m})$ (Figure 1A). The differences observed between Ocrude and OPL15 are 258 unexpected given the similarity in their composition (Table 1). On the other hand, emulsions 259 made from sunflower oils containing increasing amounts of phytosterols had comparable 260 particle size distributions, on average $2.2 \mu \mathrm{m}$ (Figure 1B). The differences in the lipid 261 composition of the oils do not explain the variability observed in the particle size

262 distributions. Therefore, for identical emulsification methods, the lipid type and quantity are 263 not the only parameters influencing the droplet size of the emulsions. Other constituents of 264 the oils, even though present in minute amount, may have altered the interaction(s) between 265 the components of the emulsion and thereby the emulsification process (e.g. tocopherols and 266 minerals such as calcium). Addition of $\alpha$-tocopherols to an emulsion made from milk lipids 267 was shown to increase the size of the emulsion droplets (Relkin, Yung, Kalnin, \& Ollivon, 268 2008). It is therefore possible that certain bioactives present in oat oil may have crystallised 269 during homogenisation, which could have led to droplet aggregation (McClements, 2012).

270 Moreover, the presence of calcium in the emulsion preparation before homogenisation has 271 been shown to influence the concentration of proteins at the surface of the emulsion droplets, 272 and thereby the droplets size (Ye \& Singh, 2001). The droplet size would also have been 273 affected by the viscosity of the oil phase (Cornec et al., 1998), which could have been 274 influenced by the different lipid compositions.

275 Microscopy images revealed that the oil bodies isolated after centrifugation formed 276 some aggregates (Figures $2 \mathrm{~B} 1$ and B2). The median oil body diameter was higher $(4.5 \mu \mathrm{m})$ 277 than the ones previously reported $(1.1 \mu \mathrm{m})$ (White, Fisk, \& Gray, 2006). This difference in 
278 size may be due to the fact that in the current study oil bodies were not all separated from

279 each other. We hypothesized that some compounds released during the extraction of the oil

280 bodies, perhaps specific to the Belinda oat variety (at least in quantity), may have interacted

281 with the lipid droplets and caused aggregation. Indeed, it has been demonstrated in rapeseed

282 that the size of the oil bodies varied between plant varieties as well as with the nature and

283 composition of phospholipids and sterols, which may affect the stability of the oil bodies

284 (Boulard et al., 2015). Fusion of the oil bodies has also been observed in oat grains when the

285 amount of oleosins embedded within the monolayer coating the oil bodies was low, in

286 particular for mature grains (Heneen et al., 2008). On the contrary, in the present study the oil

287 bodies did not appear to coalesce in the oat endosperm (Figures 2A1 and A2), but they tended

288 to flocculate once extracted from the oat matrix (Figures 2B1 and B2). This phenomenon was

289 recorded in our recent work where depletion flocculation of sunflower oil droplets was

290 induced by the $\beta$-glucan released from the oat matrix following incubation of oat flakes and

291 flour (Grundy, McClements, Ballance, \& Wilde, 2018). However, no $\beta$-glucan was detected

292 in the oil body preparation when stained with a dye specific to the polysaccharide, i.e.,

293 calcofluor white (data not shown). Similarly, starch granules were completely removed

294 during the oil body extraction and therefore not present in the final preparation as revealed by

295 staining with iodine. Some of the oil bodies may resemble starch granules in appearance, but

296 they were undeniably lipids as showed in Figure S1 of the supplementary material where all

297 the particles in the image were stained with Nile red, and therefore lipid. On the other hand,

298 toluene blue staining indicated the presence of proteins (Figure S2 of the supplementary

299 material). Therefore, it is likely that complex interactions formed between some of the oat

300 components when disturbing the oat matrix during extraction, thereby making the isolation of 301 individual oil bodies challenging. 


\subsection{Digestibility experiments using the $p H$-stat method}

\subsubsection{Lipolysis kinetics}

Despite having different emulsion droplets sizes (Figure 1A), OPL15 and Ocrude had the same lipolysis kinetics ( $p=0.970$, Figure 3A). OPL4 emulsion was digested to the same extent than as the control sunflower oil $(p=0.806)$. The purification process of the oils, and the polar lipid concentration (i.e. phospholipids and galactolipids) could have altered the interactions between their constituents in the baseline material and thereby affected their behaviour during digestion (e.g. prevent change of phase - crystallisation - at the interface). Oil bodies extracted from almond have been found to be rapidly digested when in presence of pancreatin, containing proteases and phospholipase, that can hydrolyse the oil bodies membrane allowing the lipase to easily access the triacylglycerols (Beisson et al., 2001; Grundy et al., 2016). However, in the current work, the oil bodies appeared to be the least digestible substrate $(p<0.005)$ with only $7.4 \mathrm{mmol} / \mathrm{L}$ of FFA produced compared with 10.0 and $12.8 \mathrm{mmol} / \mathrm{L}$ for Ocrude and SOs, respectively (Figure 3A). The fact that some oil bodies aggregated during their extraction from the oat groats, and that the formation of flocs were formed when they oil bodies were mixed with the digestion reagents (WPI solution, bile salts, and electrolytes; Figures $3 \mathrm{C} 1$ and $\mathrm{C} 2$ ) are likely to explain these results. Indeed, an increase in droplet size diminishes the surface area per unit volume of the lipid phase and thereby affects the ability of the lipids to be hydrolysed (Reis, Watzke, Leser, Holmberg, \& Miller, 2010). Almond oil bodies have been shown to form similar structures during gastric digestion than-to the ones observed in Figure 3C, some of the almond proteins being resistant to pepsin activity (Gallier \& Singh, 2012). Hence, the network formed by a combination of compounds (possibly proteins, phytosterols, galactolipids, saponins, and phospholipids) around, or at the vicinity, of the droplets is likely to have hindered the access of the lipase to its substrate (Chu et al., 2009; Grundy, McClements, Ballance, \& Wilde, 2018). Flocculated 
328 oil bodies could be clearly seen in Figures 3C3 and C4 confirming that these newly formed

329 structures were difficult to degrade.

330 Many compounds found in oat may be responsible for the resistance to digestion of its

331 oil and oil bodies. Given the recognised positive impact of phytosterols on lipid metabolism

332 (De Smet, Mensink, \& Plat, 2012; Bard, Paillard, \& Lecerf, 2015), we chose to investigate

333 specifically their effect on lipolysis in a more controlled way by adding increasing quantities

334 (0 to 2\%) to sunflower oil. A decrease in lipid digestibility, proportional to the concentration

335 of phytosterols in the oil, was recorded with $10.5 \mathrm{mmol} / \mathrm{L}$ of FFA produced for the oil

336 containing $2 \%$ of phytosterols (Figure $3 \mathrm{~B}$ ). Published in vitro studies examining the impact of

337 phytosterols on lipolysis are scarce. One group found that disodium ascorbyl phytostanol

338 phosphate, but not stigmastanol, was able to reduce the extent of lipid digestion possibly by

339 competing with bile salts for occupying the interface (Zhao, Gershkovich, \& Wasan, 2012).

340 In a human study, the addition of phytosterol esters to a meal did not modify lipid digestion in

341 the duodenum (Amiot et al., 2011). The authors also showed their poor solubility in mixed

342 micelles or small vesicles. The fact that the phytosterols were esterified may explain this

343 finding. However, phytosterol esters would be converted into free sterols in the human

344 duodenum via the activity of carboxyl ester hydrolase (Gleize, Nowicki, Daval, Koutnikova,

$345 \&$ Borel, 2016).

346 Overall, the reduction in the extent of lipolysis induced by the phytosterols added to

347 the sunflower oil was less important than for some of the oat materials (i.e. oil bodies, Ocrude

348 and OPL15), even though the latter contained much less phytosterols ( $2 \mathrm{~g}$ in the SOs

349 compared with an average of $\sim 0.3 \mathrm{~g}$ for the oat oils, Table 1). This implies that the diminution

350 in digestibility of lipids from oat is more likely to be due to a combination of processes, some

351 of which involving phytosterols. 


\subsubsection{Micelles characterisation}

In order to shed some light on the possible mechanism(s) behind the reduction in lipid digestibility in presence of some of the materials studied here, the micelles in the aqueous

356 phase were isolated and analysed for size and charge. The micelles are important for

357 transporting the lipolytic products away from the oil phase, and so they play a key role in the

358 lipid digestion process. Clear differences were observed in the $\zeta$-potential and size of the

359 micelles produced from either blank (control experiments without enzyme) or digested 360 samples (Figure 4). The particle size distribution of the micelles showed, for all blank 361 samples, two peaks: one around $5 \mathrm{~nm}$ and a second one around $200 \mathrm{~nm}$ (Figures 4A1 and

362 B1). Interestingly, the micelles in the blank oil bodies sample had another size peak at 11-12 $363 \mathrm{~nm}$. Following Digestion with the addition of enzymes resulted in a dramatic shift towards

364 the formation of the larger micelles of more homogeneous size $(\sim 150 \mathrm{~nm})$. For all samples, 365 apart for from the digested SOs and oil bodies, the micelle population at $\sim 5 \mathrm{~nm}$ diameter 366 completely disappeared (Figures 4A2 and B2). To investigate the effect of phytosterols, the 367 experiment was repeated in the presence of increasing concentrations of phytosterols (Figures 368 4B1 and B2). All the blank samples again showed the two populations around $5 \mathrm{~nm}$ and 200 $369 \mathrm{~nm}$. However, following the addition of the enzymes, for all samples containing phytosterols, 370 the population at $\sim 5 \mathrm{~nm}$ diameter completely disappeared, suggesting that the micellar 371 behaviour of the different oils during digestion was strongly influenced by the presence of 372 phytosterols. Regarding the $\zeta$-potential, the micelles from the oil bodies samples also had lower 374 values (-9.2 and $-18.0 \mathrm{mV}$ for blank and digested samples, respectively) compared with the 375 other materials (overall about -15.6 and $-28.6 \mathrm{mV}$ for blank and digested samples, 376 respectively) (Figures $4 \mathrm{C} 1$ and $\mathrm{C} 2$ ). The lower charge recorded for the micelles of the oil 377 bodies reflects the disparity in the initial structure, and thereby digestibility, between this 
378 complex material and the emulsions which resulted in the formation of mixed micelles of

379 different sizes and compositions. The $\zeta$-potential values of the micelles obtained from the

380 digestion of emulsions containing phytosterols are in disagreement with other studies, i.e.,

381 below -45 mV down to -65 mV (Rossi, Seijen ten Hoorn, Melnikov, \& Velikov, 2010; Nik,

382 Corredig, \& Wright, 2011). The characteristics of the emulsion (e.g. starting droplet size and

383 emulsifier) and the digestion models used may explain the discrepancy in the micelles

384 generated during lipolysis. An important observation here is the complete disappearance of

385 the $5 \mathrm{~nm}$ population following digestion of samples with any significant levels of

386 phytosterols. The smaller, $5 \mathrm{~nm}$ population is likely to be comprised mainly of simple bile

387 salt micelles, and the larger population will be mixed micelles and/or vesicles. The bile salts

388 in the small micelles can exchange rapidly with those in solution and adsorbed to the

389 interface, leading to the rapid adsorption and desorption kinetics (Parker, Rigby, Ridout,

390 Gunning, \& Wilde, 2014). This is thought to be responsible for the ability of bile salts to

391 remove lipolytic products from the interface. The disappearance of the small micelle peak

392 suggests that the equilibrium between the different populations has changed, shifting towards

393 the larger population. This indicates that bile salt micelles or free bile salts are bound up more

394 effectively into the larger structures. This could have implications for the transport and

395 absorption of lipids and lipophilic compounds if these structures are more stable, and may

396 bind or sequester lipophilic compounds more strongly.

\subsection{Impact of phytosterols on interfacial tension}

The digestibility experiments data presented above show that phytosterols have the

400 capacity of reducing to reduce the rate and extent of lipolysis. The latter process depends on

401 the "quality" of the oil/water interface, i.e., its composition and physico-chemical properties

402 (Reis, Watzke, Leser, Holmberg, \& Miller, 2010). The purpose of the interfacial experiments 
403

404

405

406

407 Furthermore, at higher phytosterol concentrations, crystalline structures were observed on the

408 surface of the oil droplets (Figure 5A2), which also affected the shape of the droplets

409 suggesting the formation of a strong, rigid structure at the interface, which could affect lipase

410 accessibility. As anticipated, bile salts alone also reduced the interfacial tension of the oil

411 droplet $(\sim 5.5 \mathrm{mN} / \mathrm{m})$, but the presence of phytosterols did not significantly affect the surface

412 tension ef when the bile salts were present (Figure 5B). Nevertheless, phytosterols occupied

413 part of the interfacial space, as illustrated by the crystalline structure forming at the edge of

414 the needle tip (red arrow in Figure 5B). Initially, it was hypothesised that the phytosterols

415 reduced the extent of FFA released due to their competition with bile salts at the interface of

416 the oil droplets. However, this was not the case as demonstrated by the fairly constant

417 interfacial tension overtime (between 5 and $5.5 \mathrm{mN} / \mathrm{m}$ ).

418 Immediately after the addition of the enzyme, the interfacial tension dropped further for up to $419 \sim 35$ min when the oil droplet detached from the needle. The bile salts from the aqueous phase 420 seemed to occupy the interface very rapidly (Figure 5B) and must have been more surface

421 active than the phytosterols, consequently the lipolysis process monitored by interfacial

422 tension was unchanged between the oils (Figure 5C). Therefore, in these experiments, the

423 phytosterols did not prevent the adsorption of the lipase/colipase onto the surface of the oil

424 droplet. The formation of lipolytic products at the interface, which would have inhibited

425 lipase activity, are likely to be responsible for the reduction in interfacial tension (Reis,

426 Watzke, Leser, Holmberg, \& Miller, 2010). Furthermore, Figure 4B2 suggested that the

427 composition of the aqueous phase, in particular the nature of the mixed micelles, may have 
428 differed in presence of oil enriched in phytosterols compared with the sunflower oil only.

429 However, the dynamic events taking place at the interface could not be specifically identified

430 with the pendant drop technique. Indeed, it is likely that the process by which phytosterols

431 impact lipolysis is time dependent. The phytosterols may affect the ability of the bile salt

432 micelles to remove the lipolytic products because the properties of the micelles change

433 following the incorporation of phytosterols. Therefore, these micelles need time to form first

434 before any effect of the phytosterols is observed. As a consequence, the kinetic curves

435 showed in Figure 3B appeared similar for all samples up to $\sim 6$ to 8 min of reaction,

436 suggesting that this may be the time required for the changes to the micelle structure to occur

437 in the presence of phytosterols.

438

439 The $\mathrm{pH}$-stat experiments measured the amount of fatty acids released into solution from the

440 oil phase following lipolysis, and demonstrated that phytosterols had some impact upon their

441 release of the FFA. However, in conjunction with other components, such as polar lipids in

442 the oat oil samples, phytosterols could have an additive effect. Furthermore, the more

443 complex interfacial structure and aggregation of the oil bodies would further complicate the

444 digestion process. The micelle behaviour is quite intriguing as this would not be detected by

445 the pH-stat measurements, but could have significant impact on the downstream fate of the

446 mixed micelles / vesicle structures. If the phytosterol were acting to stabilise these structures,

447 they could reduce both the solubilisation of lipolytic products and also the absorption of

448 lipids, bile salts and cholesterol, thus helping to explain the mechanisms underpinning the

449 health impact of phytosterols. Further work could focus on the dynamics of these micelles

450 and their bioaccessibility.

\section{4. Conclusions}


This study evaluated the impact of the composition and overall structure of different

454 lipid systems on the lipolysis process occurring in the duodenal compartment. The findings

455 from the present work revealed that the digestibility of lipids from oat relies on the degree of

456 complexity and/or purity of the oat material. Composition alone is not sufficient to explain

457 the effect that the oat compounds, albeit present in small amount, have on lipid metabolism.

458 Phytosterols appeared to play a role in the reduction in lipid hydrolysis, possibly by affecting

459 the stability and physico-chemical properties of the mixed micelles but the interactions

460 between other oat constituents and digestion agents also seem crucial. The mechanisms

461 responsible for the flocculation of the oil bodies warrant further research. In particular, it

462 would be interesting to investigate the fate of these structures during oral processing and

463 gastric digestion. Additional work would also focus on the broader matrix effects and

464 interactions between oat components that may further explain the complex mechanisms

465 underpinning the impact of oat-based products on health.

467 Acknowledgements

468 The authors would like to thank Dimitris Latousakis for his help with the GC-MS analysis.

469 This work was funded by the BBSRC project BB/R012466/1, and the BBSRC Food and Health

470 Institute Strategic Programme Grant BBS/E/F/00044418 (Quadram Institute Bioscience). RPH

471 is funded by the BBSRC Tailoring Plant Metabolism Institute Strategic Programme grant

472 BB/P012663/1 (Rothamsted Research).

474 Conflicts of interest

475 The authors are not aware of any affiliations, memberships, funding, or financial holdings that

476 might be perceived as affecting the objectivity of this work. 
478

479 480

\section{References}

Alvarez-Sala, A., Garcia-Llatas, G., Cilla, A., Barbera, R., Sanchez-Siles, L. M., \& Lagarda, M. J. (2016). Impact of lipid components and emulsifiers on plant sterols bioaccessibility from milk-based fruit beverages. Journal of Agricultural and Food Chemistry, 64(28), 5686-5691.

Amiot, M. J., Knol, D., Cardinault, N., Nowicki, M., Bott, R., Antona, C., Borel, P., Bernard, J.-P., Duchateau, G., \& Lairon, D. (2011). Phytosterol ester processing in the small intestine: impact on cholesterol availability for absorption and chylomicron cholesterol incorporation in healthy humans. Journal of Lipid Research, 52(6), 12561264.

Bard, J. M., Paillard, F., \& Lecerf, J. M. (2015). Effect of phytosterols/stanols on LDL concentration and other surrogate markers of cardiovascular risk. Diabetes and Metabolism, 41(1), 69-75.

Beisson, F., Ferte, N., Bruley, S., Voultoury, R., Verger, R., \& Arondel, V. (2001). Oil-bodies as substrates for lipolytic enzymes. Biochimica et Biophysica Acta - Molecular and Cell Biology of Lipids, 1531(1-2), 47-58.

Boulard, C., Bardet, M., Chardot, T., Dubreucq, B., Gromova, M., Guillermo, A., Miquel, M., Nesi, N., Yen-Nicolay, S., \& Jolivet, P. (2015). The structural organization of seed oil bodies could explain the contrasted oil extractability observed in two rapeseed genotypes. Planta, 242(1), 53-68.

Chen, Y., Cao, Y., Zhao, L., Kong, X., \& Hua, Y. (2014). Macronutrients and micronutrients of soybean oil bodies extracted at different pH. Journal of Food Science, 79(7), C1285-1291. 
501 Chu, B. S., Rich, G. T., Ridout, M. J., Faulks, R. M., Wickham, M. S., \& Wilde, P. J. (2009).

502

503

504

505

506

507

508

509

510

511

512

513

514 Modulating pancreatic lipase activity with galactolipids: effects of emulsion interfacial composition. Langmuir, 25(16), 9352-9360.

Cornec, M., Wilde, P. J., Gunning, P. A., Mackie, A. R., Husband, F. A., L., P. M., \& Clark, D. C. (1998). Emulsion stability as affected by competitive adsorption between an oilsoluble emulsifier and milk proteins at the interface. Journal of Food Science, 63(1), $39-43$.

Cusack, L. K., Fernandez, M. L., \& Volek, J. S. (2013). The food matrix and sterol characteristics affect the plasma cholesterol lowering of phytosterol/phytostanol. Advances in Nutrition: An International Review Journal, 4(6), 633-643.

De Smet, E., Mensink, R. P., \& Plat, J. (2012). Effects of plant sterols and stanols on intestinal cholesterol metabolism: suggested mechanisms from past to present. Molecular Nutrition and Food Research, 56(7), 1058-1072.

Ferguson, J. J. A., Stojanovski, E., MacDonald-Wicks, L., \& Garg, M. L. (2016). Fat type in phytosterol products influence their cholesterol-lowering potential: A systematic review and meta-analysis of RCTs. Progress in Lipid Research, 64, 16-29.

Gallier, S., \& Singh, H. (2012). Behavior of almond oil bodies during in vitro gastric and intestinal digestion. Food and Function, 3(5), 547-555.

Gleize, B., Nowicki, M., Daval, C., Koutnikova, H., \& Borel, P. (2016). Form of phytosterols and food matrix in which they are incorporated modulate their incorporation into mixed micelles and impact cholesterol micellarization. Molecular Nutrition and Food Research, 60(4), 749-759.

Grundy, M. M.-L., Carriere, F., Mackie, A. R., Gray, D. A., Butterworth, P. J., \& Ellis, P. R. (2016). The role of plant cell wall encapsulation and porosity in regulating lipolysis during the digestion of almond seeds. Food and Function, 7, 69-78. 
526 Grundy, M. M.-L., McClements, D. J., Ballance, S., \& Wilde, P. J. (2018). Influence of oat

527

528

529 components on lipid digestion using an in vitro model: impact of viscosity and depletion flocculation mechanism. Food Hydrocolloids, 83, 253-264.

Grundy, M. M.-L., Quint, J., Rieder, A., Ballance, S., Dreiss, C. A., Cross, K. L., Gray, R., Bajka, B. H., Butterworth, P. J., Ellis, P. R., \& Wilde, P. J. (2017). The impact of oat structure and $\beta$-glucan on in vitro lipid digestion. Journal of Functional Foods, 38(Part A), 378-388.

Gunness, P., \& Gidley, M. J. (2010). Mechanisms underlying the cholesterol-lowering properties of soluble dietary fibre polysaccharides. Food and Function, 1(2), 149-155.

Hartmann, M.-A. (1998). Plant sterols and the membrane environment. Trends in Plant Science, 3(5), 170-175.

Heneen, W. K., Karlsson, G., Brismar, K., Gummeson, P. O., Marttila, S., Leonova, S., Carlsson, A. S., Bafor, M., Banas, A., Mattsson, B., Debski, H., \& Stymne, S. (2008). Fusion of oil bodies in endosperm of oat grains. Planta, 228(4), 589-599.

McClements, D. J. (2012). Crystals and crystallization in oil-in-water emulsions: Implications for emulsion-based delivery systems. Advances in Colloid and Interface Science, 174, $1-30$.

Mel'nikov, S. M., Seijen ten Hoorn, J. W., \& Bertrand, B. (2004). Can cholesterol absorption be reduced by phytosterols and phytostanols via a cocrystallization mechanism? Chemistry and Physics of Lipids, 127(1), 15-33.

Moran-Valero, M. I., Martin, D., Torrelo, G., Reglero, G., \& Torres, C. F. (2012). Phytosterols esterified with conjugated linoleic acid. In vitro intestinal digestion and interaction on cholesterol bioaccessibility. Journal of Agricultural and Food Chemistry, 60(45), 11323-11330. 
550 Moreau, R. A., Whitaker, B. D., \& Hicks, K. B. (2002). Phytosterols, phytostanols, and their

551

552

553

554

555

556

557

558

559

560

561

562

563

564

565

566

567

568

569

570

571

572

573

574 conjugates in foods: structural diversity, quantitative analysis, and health-promoting uses. Progress in Lipid Research, 41(6), 457-500.

Nik, A. M., Corredig, M., \& Wright, A. J. (2011). Release of lipophilic molecules during in vitro digestion of soy protein-stabilized emulsions. Molecular Nutrition and Food Research, 55 Suppl 2, S278-289.

Ostlund, R. E. (2002). Phytosterols in human nutrition. Annual Review of Nutrition, 22, 533549.

Parker, R., Rigby, N. M., Ridout, M. J., Gunning, A. P., \& Wilde, P. J. (2014). The adsorptiondesorption behaviour and structure function relationships of bile salts. Soft Matter, $10(34), 6457-6466$.

Phan, S., Salentinig, S., Prestidge, C. A., \& Boyd, B. J. (2014). Self-assembled structures formed during lipid digestion: characterization and implications for oral lipid-based drug delivery systems. Drug Delivery and Translational Research, 4(3), 275-294.

Piironen, V., Lindsay, D. G., Miettinen, T. A., Toivo, J., \& Lampi, A.-M. (2000). Plant sterols: biosynthesis, biological function and their importance to human nutrition. Journal of the Science of Food and Agriculture, 80(7), 939-966.

Reis, P., Watzke, H., Leser, M., Holmberg, K., \& Miller, R. (2010). Interfacial mechanism of lipolysis as self-regulated process. Biophysical Chemistry, 147(3), 93-103.

Relkin, P., Yung, J.-M., Kalnin, D., \& Ollivon, M. (2008). Structural behaviour of lipid droplets in protein-stabilized nano-emulsions and stability of $\alpha$-tocopherol. Food Biophysics, 3(2), 163-168.

Rossi, L., Seijen ten Hoorn, J. W. M., Melnikov, S. M., \& Velikov, K. P. (2010). Colloidal phytosterols: synthesis, characterization and bioaccessibility. Soft Matter, 6(5), 928936. 
575 Ruiz-Lopez, N., Haslam, R. P., Napier, J. A., \& Sayanova, O. (2014). Successful high-level

576

577

578

579

580

581

582

583

584

585

586

587

588

589

590

591

592

593

594

595

596

597

598 accumulation of fish oil omega-3 long-chain polyunsaturated fatty acids in a transgenic oilseed crop. The Plant Journal, 77(2), 198-208.

Shewry, P. R., Piironen, V., Lampi, A. M., Nystrom, L., Li, L., Rakszegi, M., Fras, A., Boros, D., Gebruers, K., Courtin, C. M., Delcour, J. A., Andersson, A. A., Dimberg, L., Bedo, Z., \& Ward, J. L. (2008). Phytochemical and fiber components in oat varieties in the HEALTHGRAIN Diversity Screen. Journal of Agricultural and Food Chemistry, 56(21), 9777-9784.

Thies, F., Masson, L. F., Boffetta, P., \& Kris-Etherton, P. (2014). Oats and CVD risk markers: a systematic literature review. British Journal of Nutrition, 112 Suppl 2, S19-30.

von Bonsdorff-Nikander, A., Christiansen, L., Huikko, L., Lampi, A. M., Piironen, V., Yliruusi, J., \& Kaukonen, A. M. (2005). A comparison of the effect of medium- vs. long-chain triglycerides on the in vitro solubilization of cholesterol and/or phytosterol into mixed micelles. Lipids, 40(2), 181-190.

Welch, R. W. (2011). Nutrient composition and nutritional quality of oats and comparisons with other cereals. In F. H. Webster \& P. J. Wood (Eds.), Oats: chemistry and technology 2nd ed., (pp. 95-107). St Paul: American Association of Cereal Chemists, Inc (AACC).

White, D. A., Fisk, I. D., \& Gray, D. A. (2006). Characterisation of oat (Avena sativa L.) oil bodies and intrinsically associated E-vitamers. Journal of Cereal Science, 43(2), 244249.

Wolever, T. M. S., Tosh, S. M., Gibbs, A. L., Brand-Miller, J., Duncan, A. M., Hart, V., Lamarche, B., Thomson, B. A., Duss, R., \& Wood, P. J. (2010). Physicochemical properties of oat $\beta$-glucan influence its ability to reduce serum LDL cholesterol in 
humans: a randomized clinical trial. American Journal of Clinical Nutrition, 92(4),

600 723-732.

601 Ye, A., \& Singh, H. (2001). Interfacial composition and stability of sodium caseinate

602 emulsions as influenced by calcium ions. Food Hydrocolloids, 15(2), 195-207.

603 Zhao, J., Gershkovich, P., \& Wasan, K. M. (2012). Evaluation of the effect of plant sterols on 604 the intestinal processing of cholesterol using an in vitro lipolysis model. International 605 Journal of Pharmaceutics, 436(1-2), 707-710.

606 